\title{
QUEEN'S
UNIVERSITY
BELFAST
}

\section{Ultrafast charge dynamics in an amino acid induced by attosecond pulses}

Calegari, F., Ayuso, D., Trabattoni, A., Belshaw, L., De Camillis, S., Frassetto, F., Poletto, L., Palacios, A., Decleva, P., Greenwood, J. B., Martín, F., \& Nisoli, M. (2015). Ultrafast charge dynamics in an amino acid induced by attosecond pulses. IEEE Journal of Selected Topics in Quantum Electronics, 21(5), [8700512]. https://doi.org/10.1109/JSTQE.2015.2419218

Published in:

IEEE Journal of Selected Topics in Quantum Electronics

\section{Document Version:}

Peer reviewed version

Queen's University Belfast - Research Portal:

Link to publication record in Queen's University Belfast Research Portal

\section{Publisher rights}

Copyright 2016 IEEE. Personal use of this material is permitted. Permission from IEEE must be obtained for all other users, including reprinting/ republishing this material for advertising or promotional purposes, creating new collective works for resale or redistribution to servers or lists, or reuse of any copyrighted components of this work in other works

\section{General rights}

Copyright for the publications made accessible via the Queen's University Belfast Research Portal is retained by the author(s) and / or other copyright owners and it is a condition of accessing these publications that users recognise and abide by the legal requirements associated with these rights.

Take down policy

The Research Portal is Queen's institutional repository that provides access to Queen's research output. Every effort has been made to ensure that content in the Research Portal does not infringe any person's rights, or applicable UK laws. If you discover content in the Research Portal that you believe breaches copyright or violates any law, please contact openaccess@qub.ac.uk. 


\section{橉 Phé}

\section{Ultrafast Charge Dynamics in an Amino Acid Induced by Attosecond Pulses}

\begin{tabular}{|c|c|}
\hline Journal: & Journal of Selected Topics in Quantum Electronics \\
\hline Manuscript ID: & JSTQE-INV-AT2015-05635-2014 \\
\hline Manuscript Type: & Invited \\
\hline Date Submitted by the Author: & 28-Nov-2014 \\
\hline Complete List of Authors: & $\begin{array}{l}\text { Calegari, Francesca; CNR-IFN, } \\
\text { Ayuso, David; Universidad Autónoma de Madrid, Department of Chemistry } \\
\text { Trabattoni, Andrea; Politecnico di Milano, Department of Physics } \\
\text { Belshaw, Louise; Queen's University Belfast, } \\
\text { De Camillis, Simone; Queen's University Belfast, } \\
\text { Frassetto, Fabio; CNR-IFN, } \\
\text { Poletto, Luca; CNR-IFN, } \\
\text { Palacios, Alicia; Universidad Autónoma de Madrid, Department of } \\
\text { Chemistry } \\
\text { Decleva, Piero; Università di Trieste, Dipartimento di Scienze Chimiche e } \\
\text { Farmaceutiche } \\
\text { Greenwood, Jason; Queen's University Belfast, } \\
\text { Martin, Fernando; Universidad Autonoma de Madrid, Quimica } \\
\text { Nisoli, Mauro; Politecnico di Milano, Department of Physics }\end{array}$ \\
\hline Keyword: & Ultrafast optics \\
\hline
\end{tabular}




\title{
Ultrafast Charge Dynamics in an Amino Acid Induced by Attosecond Pulses
}

\author{
Francesca Calegari, David Ayuso, Andrea Trabattoni, Louise Belshaw, Simone De Camillis, Fabio \\ Frassetto, Luca Poletto, Alicia Palacios, Piero Decleva, Jason B. Greenwood, Fernando Martín, and \\ Mauro Nisoli
}

\begin{abstract}
In the past few years attosecond techniques have been implemented for the investigation of ultrafast dynamics in molecules. The generation of isolated attosecond pulses characterized by a relatively high photon flux has opened up new possibilities in the study of molecular dynamics. In this paper, we report on experimental and theoretical results of ultrafast charge dynamics in a biochemically relevant molecule, namely, the amino acid phenylalanine. The data represent the first experimental demonstration of the generation and observation of a charge migration process in a complex molecule, where electron dynamics precede nuclear motion. The application of attosecond technology to the investigation of electron dynamics in biologically relevant molecules represents a multidisciplinary work, which can open new research frontiers: those in which fewfemtosecond and even sub-femtosecond electron processes determine the fate of biomolecules. It can also open new
\end{abstract}

We acknowledge the support from the European Research Council under the ERC grants no. 227355 ELYCHE and 290853 XCHEM, from LASERLAB-EUROPE (grant agreement no. 284464, EC's Seventh Framework Programme), from European COST Action CM1204 XLIC, the MICINN Project FIS2013-42002-R, the ERA-Chemistry Project PIM2010EEC-00751, the European Grants MC-ITN CORINF and MC-RG ATTOTREND 268284, the UK's STFC Laser Loan Scheme, the Engineering and Physical Sciences Research Council (grant EP/J007048/1), the Leverhulme Trust (grant RPG-2012-735), and the Northern Ireland Department of Employment and Learning. This work was accomplished with an allocation of computer time from CCC-UAM and Mare Nostrum BSC.

F. Calegari is with the Institute of Photonics and Nanotechnologies, IFNCNR, Piazza L. da Vinci 32, 20133 Milano, Italy (e-mail: francesca.calegari@polimi.it).

D. Ayuso and A. Palacios are with the Departamento de Química, Modulo 13, Universidad Autónoma de Madrid, Cantoblanco 28049 Madrid, Spain (email:david.ayuso@uam.es; alicia.palacios@uam.es).

A. Trabattoni is with the Department of Physics, Politecnico di Milano, Piazza Leonardo da Vinci 32, 20133 Milano, Italy (e-mail: andrea.trabattoni@polimi.it).

L. Belshaw, S. De Camillis and J. B. Greenwood are with the Centre for Plasma Physics, School of Maths and Physics, Queen's University Belfast, BT7 1NN, United Kingdom (e-mail: lbelshaw01@qub.ac.uk; sdecamillis01@qub.ac.uk; J.Greenwood@qub.ac.uk).

F. Frassetto and L. Poletto are with the Institute of Photonics and Nanotechnologies, IFN-CNR, Via Trasea 7, 35131 Padova, Italy (e-mail: fabio.frassetto@pd.ifn.cnr.it; poletto@dei.unipd.it).

P. Decleva is with the Dipartimento di Scienze Chimiche e Farmaceutiche, Università di Trieste and CNR-IOM, 34127 Trieste, Italy (e-mail: decleva@univ.trieste.it).

F. Martín is with Departamento de Química, Modulo 13, Universidad Autónoma de Madrid, Cantoblanco and the Instituto Madrileño de Estudios Avanzados en Nanociencia, Cantoblanco, 28049 Madrid, Spain (e-mail: fernando.martin@uam.es).

M. Nisoli is with the Department of Physics, Politecnico di Milano and with the Institute of Photonics and Nanotechnologies, IFN-CNR, Piazza Leonardo da Vinci 32, 20133 Milano, Italy (e-mail: mauro.nisoli@polimi.it). perspectives for the development of new technologies, for example in molecular electronics, where electron processes on an ultrafast temporal scale are essential to trigger and control the electron current on the scale of the molecule.

Index Terms - Molecular physics, attosecond, femtosecond, extreme-ultraviolet (XUV) spectroscopy, high harmonics, ultrafast optics.

\section{INTRODUCTION}

U LTRAFAST DYNAMICS in molecules is at the root of a number of physical, chemical and biological processes [1]-[3]. Indeed, chemical reactions are the result of bond breaking and formation, a dynamical process that is initiated by changes in the electronic structure. The study of the dynamics associated to the nuclear motion belongs to the realm of femtochemistry, a well-established field that for more than twenty years has been able to capture and even control the nuclear motion in chemical reactions and intramolecular processes [4]. The initial step, i.e., electron rearrangement, remains to be fully understood.

Time-resolved studies on electron dynamics can shed light on the underlying mechanisms in a variety of processes such as photosynthesis, cellular respiration or electron transport along large peptides and proteins [5], [6]. Electron dynamics of those processes are induced by absorption of photons in the ultraviolet (UV) and extreme UV (XUV) range and occur in the attosecond time scale. Experimental tools and techniques able to provide such time resolution in complex molecular targets are still in their infancy [7]. The first pump-probe experiment on molecules using an isolated attosecond pulse to launch electron dynamics was reported in 2010, where electron localization was then measured in $\mathrm{H}_{2}$ and $\mathrm{D}_{2}$ using an infrared (IR) probe pulse and recorded with attosecond time resolution [8]. One year later, trains of attosecond pulses were also used to investigate how a moderately intense IR field affects the electronic dynamics induced by the XUV photoionization of hydrogen molecules [9], or to demonstrate the possibility of controlling dissociative ionization of oxygen molecules [10]. Attosecond pulse trains have been also combined with two near-IR pulses to coherently excite and control the outcome of a simple chemical reaction in $\mathrm{D}_{2}$ molecules [11]. 
Further applications of attosecond technology nowadays focus on larger and complex targets. Already in 2012, successful results have been reported on the use of a short train of attosecond pulses to explore the ultrafast dynamics in an amino acid [12]. In this work, the evolution of various ionic fragments of phenylalanine was followed in time after prompt single-photon ionization of the molecule by the XUV attosecond pulses. More recently, the authors of the present paper have reported the first experimental demonstration of charge migration in the same amino acid. It was found that this process is characterized by sub-4.5 fs oscillations appearing in the quantum yield of a specific doubly charged fragment [13].

Charge migration refers to a charge transfer mechanism occurring on a temporal scale that precedes notable nuclear motion [14], [15]. The first experimental glimpse of an extremely efficient and ultrafast charge transfer within polypeptide molecules, was reported by Schlag and coworkers [16], [17]. They showed that if an electron is selectively ionized from a chromophore at the C-terminal end of a peptide, the location of the charge can be probed by using the shift in absorption of the chromophore once charged. By employing this technique, they were able to observe a charge transfer over more than $10 \sigma$-bonds. They interpreted their experiments in terms of a very fast charge-transfer process, which can efficiently compete with fast intra-chromophoric rates. They also showed that this charge transfer was directly correlated with the properties of individual amino acids.

The ultrafast dynamics of charge transfer processes initiated in complex biologically relevant molecules by sudden photoionization have been investigated in various theoretical papers [14], [15], [18], [19]. It was suggested that if an electron is suddenly removed from a molecular orbital, the molecule will be in a superposition of electronic states of the radical cation. The temporal evolution of this electronic wavepacket, producing charge oscillations, has been referred to as charge migration to distinguish it from charge transfer mediated by nuclear motion. For example, a hole generated by prompt ionization of the highest-occupied molecular orbital (HOMO) of the tetrapeptide Trp-Leu-Leu-Leu (Trp and Leu are the amino acids Tryptophan and Leucine, respectively) and localized on the Trp chromophore migrates to the $\mathrm{N}$ terminus (or amino-terminus) of the peptide in 0.75 fs [15]. Direct experimental access to such sub-femtosecond processes is mandatory to image electronic dynamics in complex molecules and requires of attosecond measurement techniques.

In this work, we report experimental measurements and a detailed theoretical analysis of the first application of isolated attosecond pulses to phenylalanine. In Section II, the pumpprobe experimental setup is described. Experimental results are discussed in Section III, where we first present the mass spectrum of phenylalanine produced upon photoionization by isolated attosecond pulses, followed by the pump-probe measurements that reveal the signature of ultrafast charge migration along the molecular skeleton. The theoretical data and interpretation are given in Section IV. Finally, Section V presents a summary of the most significant results.

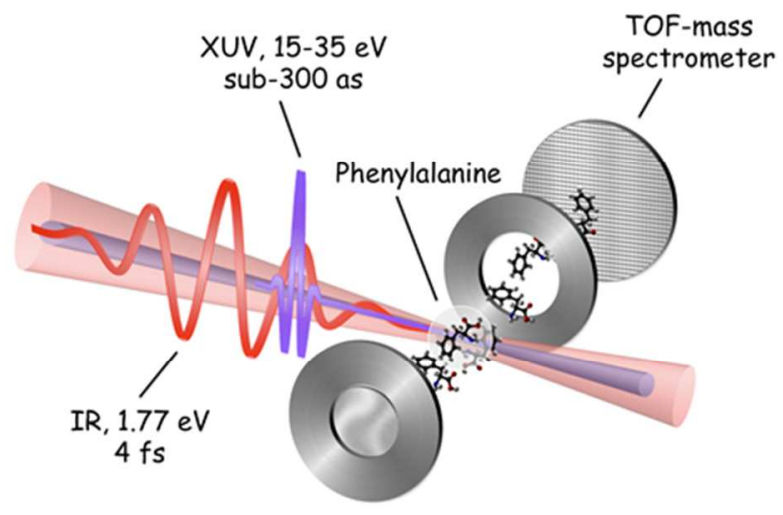

Fig. 1. Experimental setup for the pump-probe measurements showing the time-of-flight mass spectrometer used in the experiments.

\section{EXPERIMENTAL SETUP}

For the experiments a Ti:sapphire laser system was used (Femtopower PRO HE CEP, Femtolasers) with the following characteristics: 6-mJ pulse energy, 25-fs pulse duration and 1$\mathrm{kHz}$ repetition rate. The carrier-envelope phase (CEP) was stabilized by two feedback loops: a fast loop acting on the oscillator and a slow loop acting on a glass wedge placed in the stretcher before the preamplifier. The residual CEP fluctuation was about $200 \mathrm{mrad}$ (rms), measured in-loop with a $f$-to- $2 f$ interferometer using an integration time of $1 \mathrm{~ms}$. We have then compressed the pulses using the hollow-fiber compression technique [20], [21] in combination with a broadband chirped-mirror dispersive delay line. Due to the high energy of the laser pulses, the pressure gradient scheme has been employed [22]. We used a 1-m-long hollow fiber with an inner diameter of $320 \mu \mathrm{m}$, filled with helium: the pressure at the input of the fiber was kept to $<5$ mbar and 2 bar at the output. The beam position at the input of the hollow fiber was actively stabilized [23]. A pulse energy of $3.3 \mathrm{~mJ}$ was obtained at the output with no gas in the fibre, and $2.3 \mathrm{~mJ}$ with gas. After 8 reflections on the chirped mirrors, pulses as short as 4 fs were achieved. The pulse duration was measured by using a single-shot Self-Referenced Spectral Interferometry method [24] implemented with Cross-Polarized Wave (XPW). By using a novel single-shot interferometric technique, allowing in situ measurement of intensity-dependent phase changes experienced by ultrashort laser pulses upon nonlinear propagation, we have demonstrated that CEP stability is substantially preserved by the hollow fiber compression technique [25].

The visible/near infrared (VIS/NIR) beam was divided into two portions by using an ultrabroadband beam splitter with $30 \%$ reflection. The transmitted beam part was focused by a $1-$ $\mathrm{m}$ radius of curvature mirror into a $3-\mathrm{mm}$-thick cell filled with xenon at static pressure to produce extreme ultraviolet (XUV) radiation by high-order harmonic generation (HHG). Isolated attosecond pulses, with energy in the nanojoule range, were produced by employing the ionization gating technique, which 
is a temporal gating scheme based on sub-cycle ionization dynamics in a gas cell driven by few-optical-cycle pulses with above-saturation intensity and stable CEP [26], [27]. A 100nm-thick aluminium filter was used to filter out the fundamental radiation and the low order harmonics. An indium foil has been also used in a few measurements described in the text. The temporal duration of the XUV pulses ( $290 \pm 20$ as) was measured by using the Frequency Resolved Optical Gating for Complete Reconstruction of Attosecond Bursts (FROG CRAB) technique [28], [29]. The remaining part of the VIS/NIR beam was collinearly recombined with the XUV beam by using a mirror with a central hole. The temporal delay between VIS/NIR and XUV pulses was adjusted with attosecond resolution by using a piezoelectric translation stage. The pump and probe pulses were collinearly focused into the mass spectrometer, as shown in Fig. 1, by using a gold-coated toroidal mirror, with unit magnification, which provided an almost aberration-free image of the XUV source with negligible temporal smearing of the attosecond pulses [30]. The peak intensity of the VIS/NIR probe pulse in the sample was about $5 \times 10^{12} \mathrm{~W} / \mathrm{cm}^{2}$. The spectrum of the XUV radiation was measured by using a high-resolution flat-field soft X-ray spectrometer [30].

Gas-phase molecules were produced by employing the laser induced thermal desorption method. Phenylalanine was deposited on a $10-\mu$ m-thick stainless steel foil, mounted onto the repeller electrode of a time of flight (TOF) mass spectrometer [31]. To evaporate the sample, the reverse side of the foil was irradiated with a $\mathrm{CW}$ diode laser operating at a wavelength of $960 \mathrm{~nm}$, with a spot diameter of $6 \mathrm{~mm}$ and power in the range $0.3-0.4 \mathrm{~W}$. The distance from the foil to the focal point of the ionizing laser pulses was approximately $3 \mathrm{~mm}$.

The temperature of the sample was estimated by assuming equilibrium heat conduction along the foil to the repeller electrode and that $30 \%$ of the incident radiation was absorbed by the foil at this wavelength [32]. Radiative heat losses were negligible for the calculated temperatures. For the lowest laser power used, the temperature at the centre of the foil was estimated to be $430 \mathrm{~K}$ and about $410 \mathrm{~K}$ at a radius of $2 \mathrm{~mm}$.

\section{EXPERIMENTAL RESULTS}

\section{A. Fragmentation Results}

We investigated the phenylalanine molecule, one of the essential amino acids for human life. It is an $\alpha$-amino acid, consisting in a central carbon atom ( $\alpha$ carbon), linked to an amine $\left(-\mathrm{NH}_{2}\right)$ group, a carboxylic group $(-\mathrm{COOH})$, a hydrogen atom and a side chain (-R). The side chain is specific for each amino acid and for phenylalanine is formed by a methylene ($\mathrm{CH}_{2}-$ ) group ( $\beta$ carbon) terminated by a phenyl ring. We have chosen it as a model molecule for charge migration because it contains two electron-acceptor sites with approximately the same binding energy located on the phenyl and amine groups, and separated by two singly bonded carbon atoms. The molecule was first irradiated with the XUV attosecond pulse

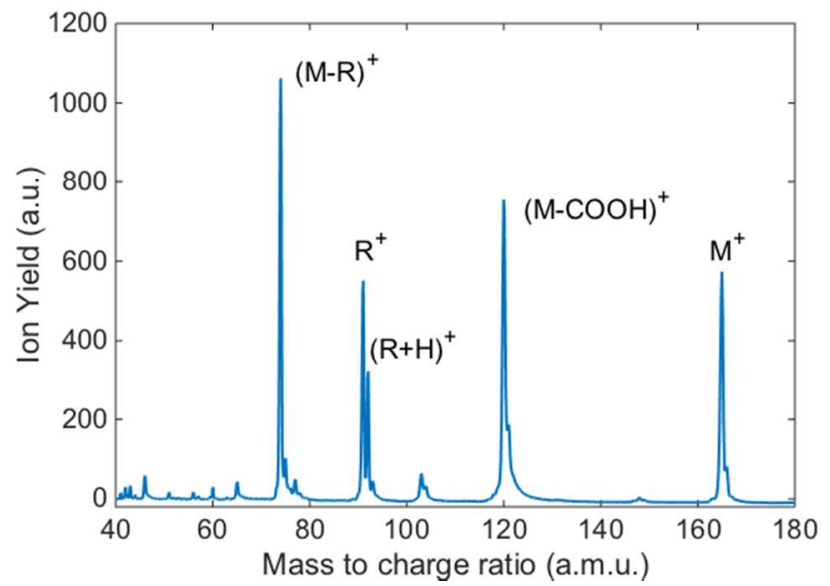

Fig. 2. Mass spectrum from ionization of phenylalanine by an XUV pulse. $\mathrm{M}$ stands for the parent ion and $\mathrm{R}$ for the side chain group.

which has a photon energy bandwidth of $\sim 19 \mathrm{eV}$, from $16 \mathrm{eV}$ to $35 \mathrm{eV}$. The parent and fragment ions produced were then extracted into the TOF spectrometer for mass analysis. Figure 2 shows the measured mass spectrum, where the main contributions correspond to the parent ion $\mathrm{M}^{+}$(165 Da), loss of the carboxyl group yielding the immonium ion $(\mathrm{M}-\mathrm{COOH}$ at $120 \mathrm{Da}$ ), and breakage of the $\mathrm{C} \alpha-\mathrm{C}_{\mathrm{b}}$ bond with the charge residing on the amine (M-R at $74 \mathrm{Da})$ or phenyl groups $(\mathrm{R}$ at 91). We observe a small peak with a mass to charge ratio $m / q=60$, which is due to the doubly charged immonium ion. The XUV pulse is capable of ionizing all valence and some inner shell orbitals, resulting in a wide range of fragment ions. It was observed that using only the 4-fs VIS/NIR pulses to ionize the molecule produced predominantly parent ions in the mass spectrum.

\section{B. Pump-Probe Measurements}

The temporal evolution of the ionic fragments produced in phenylalanine has been investigated by means of two-color pump-probe measurements, using attosecond pump pulses and 4-fs VIS/NIR probe pulses at a variable temporal delay. To obtain the zero of the experimental data we have removed the 100-nm-thick aluminium filter and we have measured as a function of time the fragment yields produced by the VIS/NIR-pumpVIS/NIR-probe interaction. This defines our zero time position to an accuracy better than $1 \mathrm{fs}$.

In a previous work [12] we observed dynamics in a number of fragments with a time constant of $80 \pm 20 \mathrm{fs}$, which we attributed to an internal conversion process. In this work, which has much better statistics, the fractional yields of nearly all the singly charged fragments vary as a function of pumpprobe delay. However, there is a marked difference when the XUV beam is filtered with an aluminium foil, generating an XUV spectrum from 16 to $35 \mathrm{eV}$, compared to an indium foil, which produces a XUV spectrum with a $3-\mathrm{eV}$ (full-width at half maximum) peak centred around $15 \mathrm{eV}$, followed by a broad and weak spectral component extending up to $25 \mathrm{eV}$. This is demonstrated in Fig. 3 for the fragment $m / q=28$ $\left(\mathrm{NH}_{2} \mathrm{C}\right)$. It can be seen that in the case of XUV pulses transmitted by the aluminium filter (see Fig. 3(a)), the 
fragment yield increases with pump-probe delay, with a time constant of $80 \pm 2$ fs. Other fragments also increase with time constants in the range from 50 fs to $100 \mathrm{fs}$ at the expense of the parent $(m / q=165)$ and immonium ions $(m / q=120)$ which decrease. By contrast, in the case of XUV pulses transmitted by the indium filter (see Fig. 3(b)), a sudden increase in the yield of the fragment $m / q=28$ can be observed with no subsequent dynamics. This result suggests that when only a valence electron can be ionized (XUV pulse filtered by indium), no temporal dynamics of the singly charged ions are observed, but the presence of hole in the valence shell allows the VIS/IR pulse to be absorbed. For instance, it is known that absorption by the phenyl chromophore shifts from the
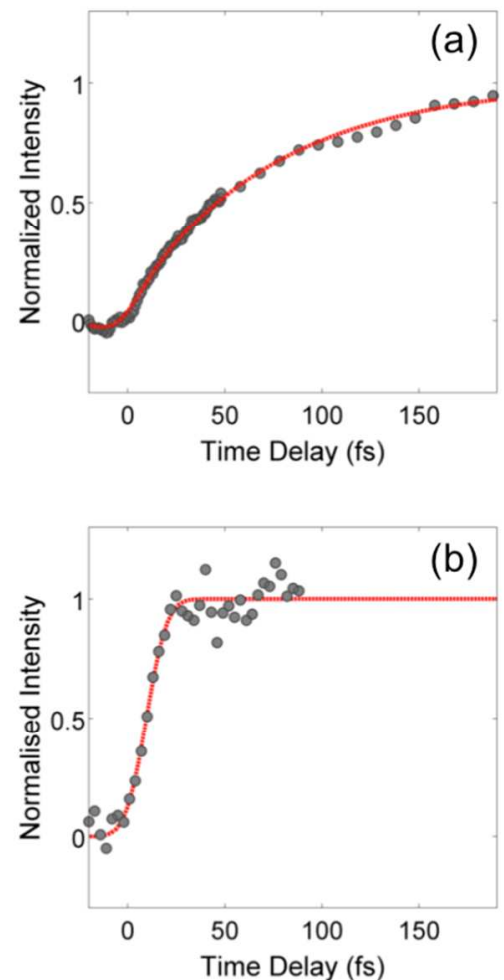

Fig. 3. Normalized yield of fragment $m / q=28$, as a function of pump-probe delay for an XUV pump pulse transmitted by an aluminium foil (a) and an indium foil (b). In (a) the data has been fitted with the convolution of a Gaussian function (4-fs FWHM) with the function $y=y_{0}+b[1-\exp (-t / \tau)]$. In (b) the data has been fitted with the convolution of a Gaussian function with the step function.

ultraviolet to the green when there is a hole in the highest occupied molecular orbital [17]. This increases the yield of smaller fragments when the XUV pulse precedes the VIS/IR pulse with a concomitant reduction in parent and larger fragment ions. When an inner valence orbital is ionized (by the XUV pulse filtered with aluminium), there is initially no resonant absorption of the probe but an internal conversion to a lower electronic state generates a hole in the valence shell opening up absorption of the probe and hence further fragmentation. The measured timescale of 50-100 fs is compatible with an internal conversion mechanism as it is mediated by nuclear motion on potential energy surfaces.

On the other hand, the immonium dication $(\mathrm{m} / \mathrm{q}=60)$ shows a faster temporal evolution, as shown in Fig. 4, where the data

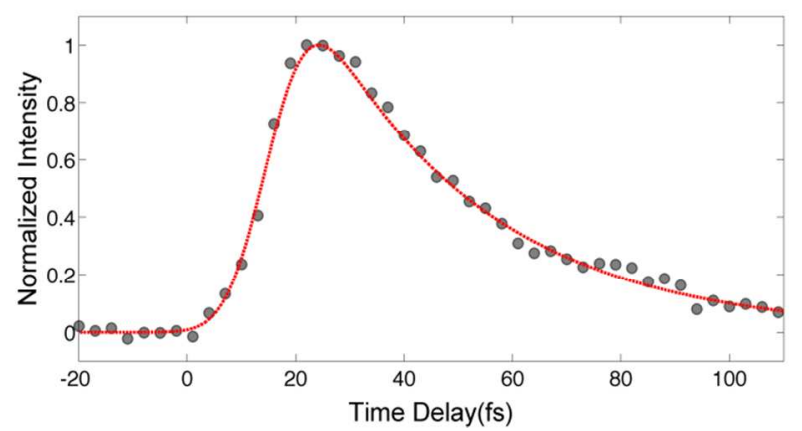

Fig. 4. Yield of doubly charged immonium ion $(m / q=60)$ as a function of pump-probe delay. The dotted line is the convolution fitting function $F(t)$ described in the text.

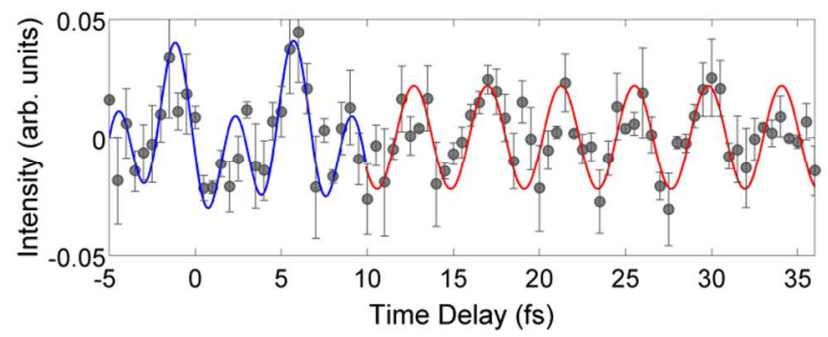

Fig. 5. Dots correspond to the difference between the experimental data and the fitting curve $F(t)$. Error bars show the standard error of the results of four measurements. Blue curve is a fitting curve obtained as the sum of two sinusoidal functions, red curve is a single sinusoidal fitting curve, as described in the text.

points have been acquired by using 3-fs step in pump-probe time delay. The experimental data has been fitted by the convolution, $F(t)$, of a Gaussian pulse of 4-fs full-width at half maximum (FWHM), with the following function:

$$
R(t)=A\left(e^{-t / \tau_{1}}-e^{-t / \tau_{2}}\right)
$$

where $\tau_{1}=10 \pm 2$ fs and $\tau_{2}=25 \pm 2$ fs. Upon decreasing the delay-step between pump and probe pulses from 3 fs to $0.5 \mathrm{fs}$, an oscillation of the dication yield is clearly visible in the pump-probe measurement. Figure 5 displays the measured data, acquired with delay-step between pump and probe pulses of $0.5 \mathrm{fs}$, after subtraction of the fitting curve $F(t)$ (assuming $\tau$ ${ }_{1}=10 \mathrm{fs}$ and $\tau_{2}=25 \mathrm{fs}$ ).

As reported in [13], we have performed a Fourier analysis of the experimental data. Time-dependent Fourier transforms have been calculated by using a sliding Gaussian window function

$$
g\left(t-t_{d}\right)=\exp \left[-\left(t-t_{d}\right)^{2} / t_{0}^{2}\right]
$$

with $t_{0}=10 \mathrm{fs}$ and peak at $t_{d}$ (gate delay time). As shown in Fig. 6 at short pump-probe delays $(t<10 \mathrm{fs})$ two main frequency components are present, around $0.14 \mathrm{PHz}$ and 0.3 $\mathrm{PHz}$. Therefore in this temporal window the pump-probe data have been fitted by the sum of two sinusoidal functions:

$$
S(t)=A_{1} \sin \left(2 \pi v_{1} t+\phi_{1}\right)+A_{2} \sin \left(2 \pi v_{2} t+\phi_{2}\right)
$$

The calculated frequencies are: $0.14 \mathrm{PHz}$ (lower and upper 
confidence bounds: $0.12 \mathrm{PHz}$ and $0.158 \mathrm{PHz}$, respectively) and $0.293 \mathrm{PHz}$ (lower and upper confidence bounds: 0.281 $\mathrm{PHz}$ and $0.304 \mathrm{PHz}$, respectively). At longer pump-probe delays (in the range from $10 \mathrm{fs}$ to $40 \mathrm{fs}$ ) a strong and broad peak around $0.24 \mathrm{PHz}$ is visible in the Fourier spectrogram. For this reason the data have been fitted with a sinusoidal function of frequency $0.234 \mathrm{PHz}$ (oscillation period of $4.3 \mathrm{fs}$ ), with lower and upper confidence bounds of 0.229 and 0.238 $\mathrm{PHz}$, respectively.

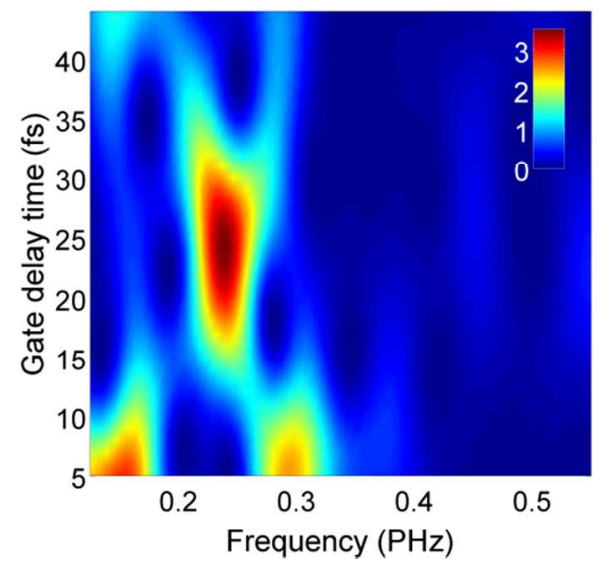

Fig. 6. Fourier sliding-window analysis of the experimental data.

\section{THEORETICAL METHOD AND DisCUSSION}

We have then performed numerical simulations in order to analyse the ultrafast charge evolution after attosecond excitation. Due to the low intensity of the attosecond pump pulses used in the experiment, time-dependent first-order perturbation theory has been employed for the calculation of the ionization amplitudes at the end of the pulse $(t=T)$ :

$$
c_{\alpha \varepsilon l m}(T)=-i\left\langle\Psi_{\alpha l m}(\varepsilon, \vec{r})|\vec{e} \cdot \vec{r}| \Psi_{0}(\vec{r})\right\rangle \int_{-\infty}^{T} E(t) e^{i\left(E_{\alpha}+\varepsilon-E_{0}\right) t} d t
$$

where $\Psi_{0}$ is the all-electron (called $N$-electron) ground state of phenylalanine with energy $E_{0}, \Psi_{\alpha l m}(\varepsilon)$ is the $N$-electron continuum state, describing a photoelectron ejected from the $\alpha$ molecular orbital with kinetic energy $\varepsilon$ and angular quantum numbers $l$ and $m(|m| \leq l), E_{\alpha}$ is the energy of the corresponding cationic species and $E(t)$ is the electric field of the XUV pulse polarized along the $\vec{e}$ direction. The $\Psi_{0}$ and $\Psi_{\alpha l m}(\varepsilon)$ wave functions have been evaluated in the framework of the fixed-nuclei approximation by using the static-exchange density functional theory (DFT) [33] method, which makes use of the Kohn-Sham DFT to describe molecular bound states and of the Galerkin approach to evaluate continuum wave functions in the field of the corresponding Kohn-Sham density. The LB94 functional [34] has been employed to account for exchange and correlation effects. In order to obtain reliable values of the ionization energies $E_{\alpha}$, we have employed the VWN [35] local density approximation (LDA) functional within the Slater transition state procedure [36] using the ADF commercial program [37], [38] with a TZP basis set (taken from the ADF library). Bound and continuum states are evaluated in a multicentric basis set built with products of B-splines and spherical harmonics, as widely described in previous works [39], [40]. We use a large onecenter expansion (OCE) placed at the center of mass of the molecule to accurately describe the asymptotic behavior of the continuum states. To improve the convergence of the calculation for the description of the more localized bound states, the OCE is supplemented with $\mathrm{N}$ (number of atoms) small non-overlapping off-center expansions located at the nuclear positions. For phenylalanine, we obtain converged results using as radial parameters a OCE with a 30 a.u. radius and 23 off-center expansions extended up to 1 a.u. of radius. Because of the large size and the lack of symmetry in the phenylalanine molecule, we have included an angular expansion of 484 spherical harmonics (equivalently, angular momentum $l \leq 20$ in Eq. 4). The initial guess for the electronic density was generated with ADF [37], [38], and the molecular geometries of the different conformers were optimized at the DFT-B3LYP [41], [42] level with the 6-311+g(3df,2p) basis set employing Gaussian09 computational package [43].

The electronic density of the $(N-1)$-electron cation can be calculated as [44], [45]

$$
\begin{gathered}
\rho_{\text {ion }}(\vec{r}, t)=\sum_{\alpha}\left(\sum_{\substack{\alpha^{\prime} \\
\alpha^{\prime} \neq \alpha}} \gamma_{\alpha^{\prime} \alpha^{\prime}}^{\text {ion })}\right) \varphi_{\alpha}^{2}(\vec{r})+ \\
-\sum_{\substack{\alpha \alpha^{\prime} \\
\alpha^{\prime} \neq \alpha}} \gamma_{\alpha \alpha^{\prime}}^{(\text {ion })} e^{i\left(E_{\alpha^{\prime}}-E_{\alpha}\right) t} \varphi_{\alpha}(\vec{r}) \varphi_{\alpha^{\prime}}(\vec{r})
\end{gathered}
$$

where $\varphi_{\alpha}(\vec{r})$ is the $\alpha$ molecular orbital and $\gamma_{\alpha \alpha^{\prime}}^{(\text {ion })}$ is the reduced density matrix element defined as:

$$
\gamma_{\alpha \alpha^{\prime}}^{(\text {ion })}=\sum_{l, m} \int c_{\alpha l m}(\varepsilon) c_{\alpha^{\prime} l m}^{*}(\varepsilon) d \varepsilon
$$

We have neglected the interaction of the photoelectron with the remaining $(N-1)$-electron cation. The hole density is given by the difference between the electronic density of the neutral molecule, which does not depend on time, and the electronic density of the ion, $\rho_{\text {hole }}(\vec{r}, t)=\rho_{\text {neutral }}(\vec{r})-\rho_{\text {ion }}(\vec{r}, t)$, and can be written as follows:

$$
\begin{gathered}
\rho_{\text {hole }}(\vec{r}, t)=\sum_{\alpha}\left(1-\sum_{\substack{\alpha^{\prime} \\
\alpha^{\prime} \neq \alpha}} \gamma_{\alpha^{\prime} \alpha^{\prime}}^{(\text {ion })}\right) \varphi_{\alpha}^{2}(\vec{r})+ \\
+\sum_{\substack{\alpha \alpha^{\prime} \\
\alpha^{\prime} \neq \alpha}} \gamma_{\alpha \alpha^{\prime}}^{(\text {ion })} e^{i\left(E_{\alpha^{\prime}}-E_{\alpha}\right) t} \varphi_{\alpha}(\vec{r}) \varphi_{\alpha^{\prime}}(\vec{r})
\end{gathered}
$$

where

$$
\rho_{\text {neutral }}(\vec{r})=\sum_{\alpha} \varphi_{\alpha}^{2}(\vec{r})
$$




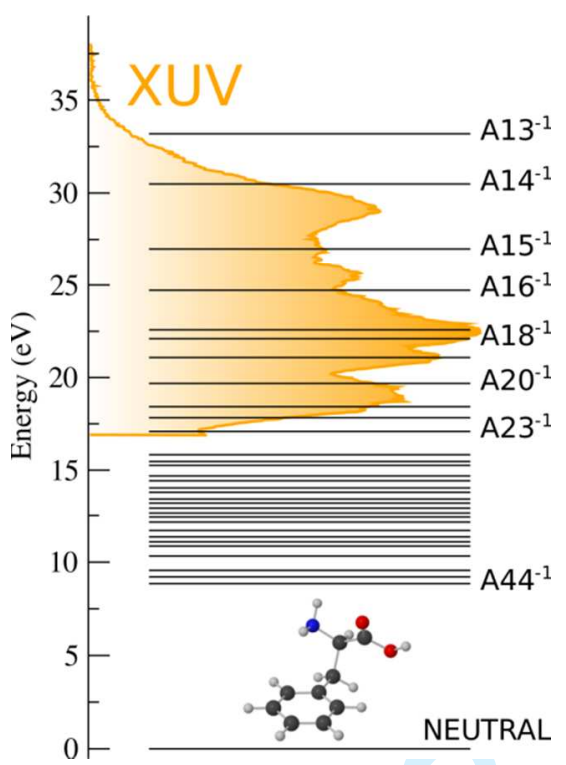

Fig. 7. Ionization potentials for the most abundant conformer of the phenylalanine molecule. The labels "An" denote the molecular orbital from which the electron is removed. The spectrum of the attosecond pulses used in the experiment and in the calculations is represented by a thick orange curve lying over a shaded area. All ionic states below the largest absorbed photon energy $(35 \mathrm{eV})$ are being populated.

Figure 7 shows the calculated ionization energies of all open channels for the most abundant conformer of phenylalanine, according to previous theoretical work [46]. These energies are approximately given by the Kohn-Sham orbital energies resulting from the VWN-transition state calculations. For a given photon energy, all channels below that energy will be populated. We are thus creating a wave packet containing all ionic states with ionization potentials below $35 \mathrm{eV}$. As illustrated in Fig. 7, $35 \mathrm{eV}$ is the largest photon energy contained within the bandwidth of the attosecond pulses used in the experiment. Their relative contributions to the total wave packet are given by both the pulse profile and the dipole couplings from the ground state of the neutral.

In order to test the validity of our description of the ionization process, we have first calculated the photoelectron spectra of phenylalanine for photon energies of 21.2 and 45 $\mathrm{eV}$, by using the computed dipole transition matrix elements and ionization potentials, and we have compared our results with synchrotron [47] and $\mathrm{He}(\mathrm{I})$ [48], [49] radiation experiments. We have convoluted our infinite resolution lines with a Gaussian function of $0.3-\mathrm{eV}$ width at half maximum to account for the vibrational broadening and experimental energy resolution, which is rather limited in this and earlier experiments (the experiments cannot resolve the individual peaks). The comparison between theory and experiment is shown in Fig. 8. As can be seen, the agreement is reasonably good. We notice however that the experiment of Plekan et al. was performed at a photon energy of $100 \mathrm{eV}$, which is substantially higher than ours. Other two earlier experiments were performed at a photon energy of $21.2 \mathrm{eV}$ [48], [49]. Our results are in better agreement with the most recent

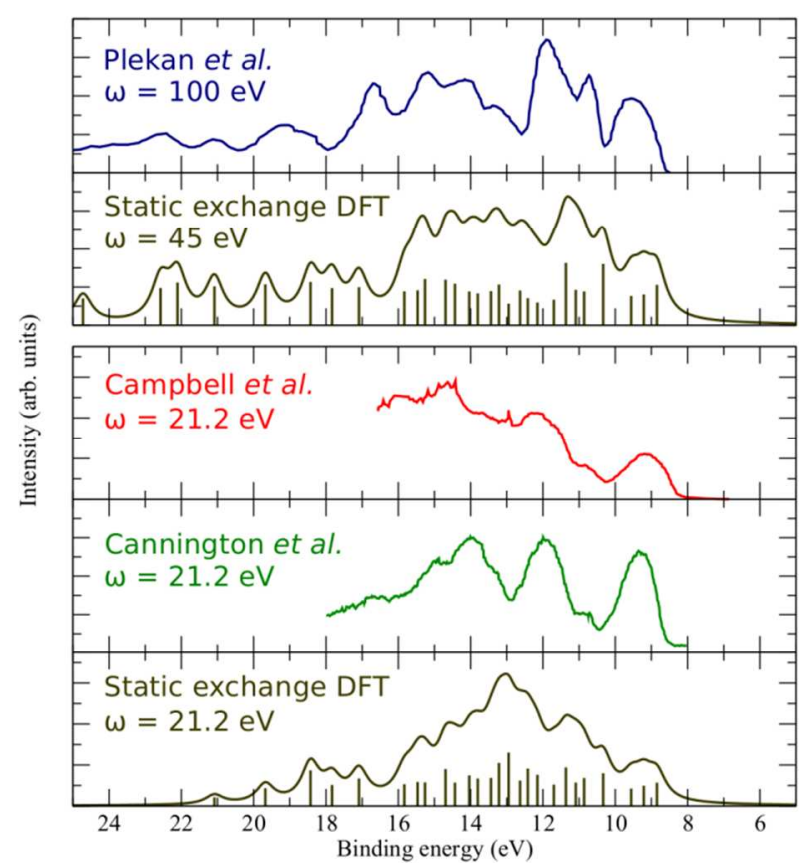

Fig. 8. Comparison between calculated and experimental photoelectron spectra of phenylalanine.

experiment, especially for binding energies below $15 \mathrm{eV}$, which includes the most relevant cationic states in the hole dynamics reported in the present work.

The electronic wave packet generated by the attosecond pulse was then calculated from the ionization amplitudes, by using the experimental frequency spectrum of the pulse (shown in Fig. 7). Time zero in the simulation corresponds to the end of the attosecond pulse and the wavepacket evolution is calculated for positive times immediately after the prompt ionization. The evolution of the electronic wave packet was then evaluated by using a standard time-dependent density matrix formalism [50], in which the system is described by a sum of single-particle Hamiltonians. Since in this work we consider ionization from the ground state, which has a pronounced single-determinant character, the effects related to 2-holes-1-particle ( $2 \mathrm{~h} 1 \mathrm{p})$ states are expected to be much smaller. In ground state photoionization, $2 \mathrm{~h} 1 \mathrm{p}$ states are nevertheless not negligible in inner-valence shell ionization, as has been extensively studied by Cederbaum and coworkers [51], but they are of minor importance in outer-valence ionization, which is the dominant process in our work. The latter conclusion is supported by photoelectron spectroscopy experiments in this and similar molecules, in which satellite lines associated to $2 \mathrm{~h} 1 \mathrm{p}$ states are always much weaker that those associated to $1 \mathrm{~h}$ states. It is also supported by new theoretical calculations that we have carried out for glycine [52], in very good agreement with the results obtained by Kuleff et al. [53]. Therefore, in the range of photon energies leading to the cationic states relevant for the observed dynamics, we can safely conclude that $2 \mathrm{~h} 1 \mathrm{p}$ states do not play a significant role.

Every ionic state is related to a single Slater determinant 
with doublet spin multiplicity. However, this single determinant is not built from Hartree-Fock (HF) orbitals but from Kohn-Sham (KS) orbitals arising from DFT calculations. In complex non symmetric molecules it often happens that there is a kind of "orbital rotation" upon ionization, expressed as a mixing of HF orbitals. This is reported e.g. in the paper by Lünnemann et al. [19]. However, this does not mean a breakdown of the one particle (1h) approximation for such states, just that the Dyson orbital, still of norm close to 1 , is a linear combination of ground state HF orbitals. Since some correlation is built in the KS orbitals, they are generally closer to the correct Dyson orbitals than the HF ones. As mentioned above, this has been tested by reproducing the results by Kuleff et al. [53] for glycine outer valence ionizations [52]. The nature of the Dyson orbital can only be probed by evaluating dynamical properties, like photoionization cross sections, or electron momentum spectroscopy. This is additionally supported by the vast number of published studies of photoionization cross sections performed with the present approach, also in molecules of comparable complexity [54][63].

Due to the random orientation of the phenylalanine molecule, we calculated the hole dynamics resulting from excitation by pulses with the electric field polarized along three orthogonal directions, and the results were then averaged. Although no electron selection rules strictly apply, due the lack of global symmetry elements, of course

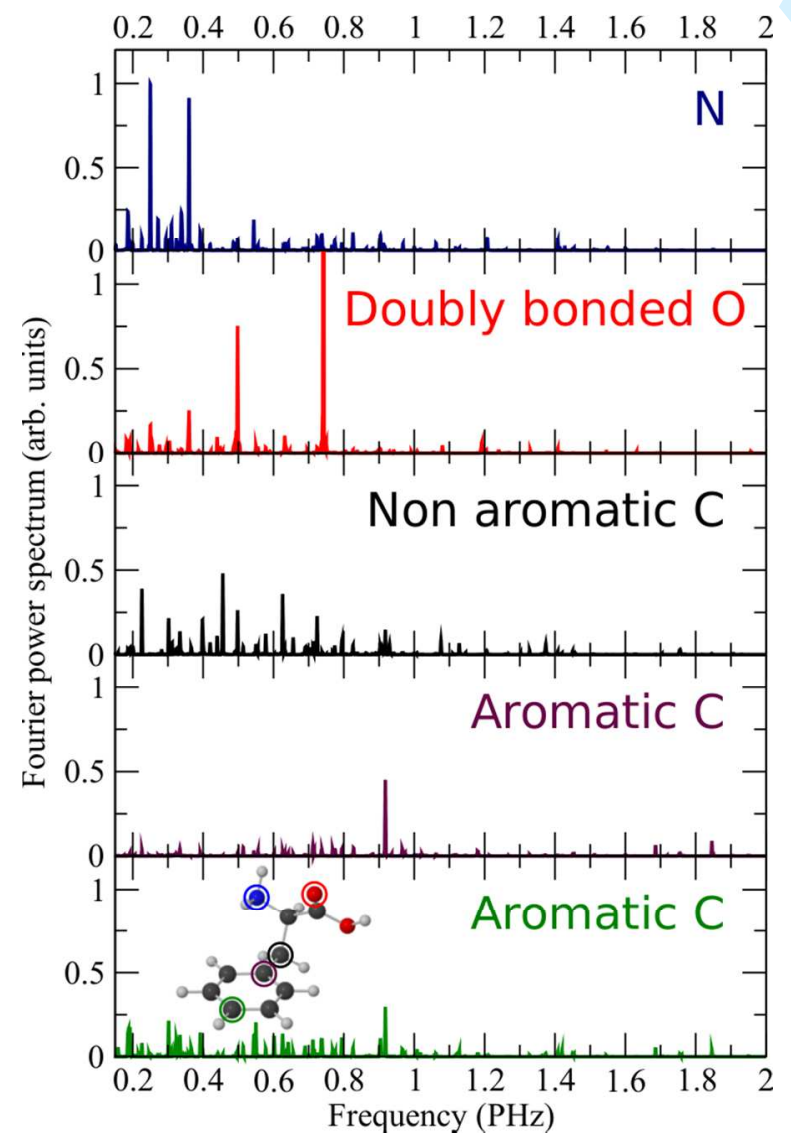

Fig. 9. Fourier power spectra of the calculated hole density integrated over various atoms of the phenylalanine molecule for the most abundant conformer. approximate local symmetries will apply, depending on the localization of the initial orbital. E.g., for an initial orbital of mainly $\sigma$ ring character, a parallel polarization excites it to $\sigma$ ring states, while a perpendicular one drives it to $\pi$ final states, and similar considerations apply to initial orbitals mainly localized on other moieties $\left(-\mathrm{NH}_{2},-\mathrm{COOH}\right.$, etc). This explains why the results obtained for the different polarization directions, $x, y$ and $z$, are different.

We note that, in the particular case considered in this work, the Lebedev sampling method to average over molecular orientation [64], would lead to the same final result. Indeed, the reduced density matrix elements $\gamma_{\alpha \alpha^{\prime}}$ (reported in Eq. 6) can be written as a function of the electron ejection direction $\left(\Omega_{\mathrm{e}}\right)$ and the orientation of the molecule $\left(\Omega_{\mathrm{n}}\right)$. For randomly oriented molecules and non-resolved electron ejection angles, one would have to integrate over both $\Omega_{\mathrm{e}}$ and $\Omega_{\mathrm{n}}$. However, this integration would only affect the $\gamma_{\alpha \alpha^{\prime}}$ terms in Eq. 7, which contain products of amplitudes with the same angular momentum $l$, but different $\alpha$ and $\alpha$. So, if the symmetry of the channels $\alpha$ and $\alpha^{\prime}$ is the same for all states (as in phenylalanine), the algebra to integrate the angular part of $\gamma_{\alpha \alpha^{\prime}}$ is exactly the same as the usual one to derive integrated cross sections (see [65] and references therein). This means that, in practice, it is enough to average the results obtained for three arbitrary but orthogonal orientations.

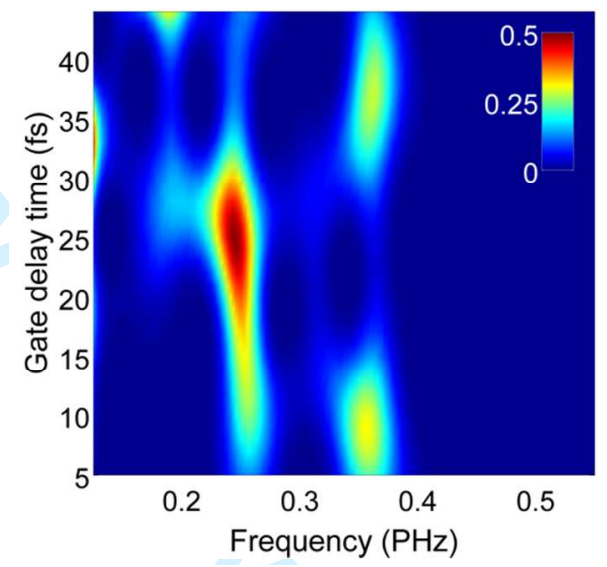

Fig. 10. Fourier sliding-window analysis of the calculated temporal evolution of hole density integrated over the amine group for the most abundant conformer of phenylalanine.

We have integrated the hole density around particular portions of the molecule. Figure 9 shows the Fourier power spectra of the calculated hole density over various atoms of the molecule: the nitrogen atom of the amine group, the double bonded oxygen atom of the carboxylic group, two carbon atoms of the phenyl group and a non-aromatic carbon atom. Beating frequencies in agreement with the experimental observations were observed when the charge density was integrated around the amine group. The hole densities at different positions in the molecules do not show clear and clean frequency components (with the exception of the doubly bonded $\mathrm{O}$ atom in the carboxyl group, which however cannot be observed in the doubly-charged immonium fragment, which results from the loss of the carboxyl group). 


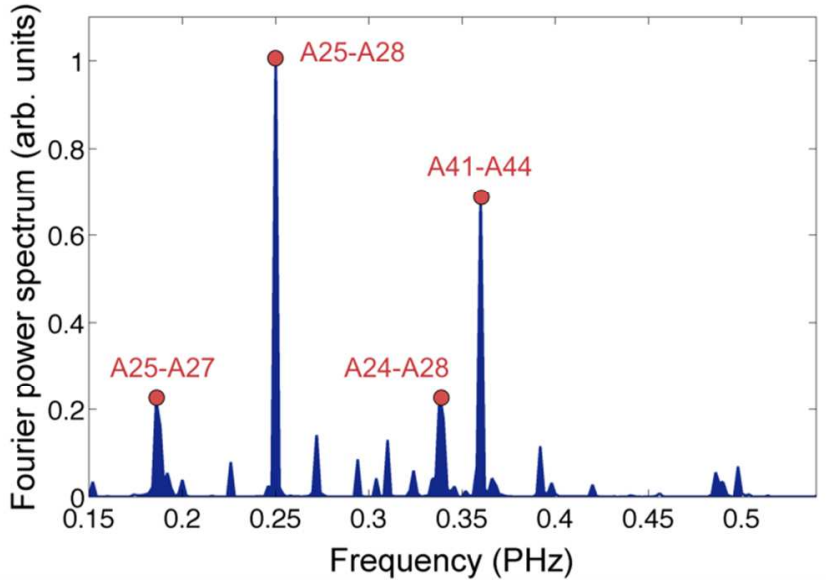

Fig. 11. Fourier power spectra of the hole density integrated over the amine group for the most abundant conformer of phenylalanine; we have identified the ionic states that are responsible for the most important beatings.

We note that the VIS/NIR probe pulse is not locally absorbed only by the amine group, but also by other sites of the molecule. However, the simulations indicate that the periodic modulations observed in the experiment are mainly related to the absorption of the probe pulse by the amine group. The charge dynamics around the amine group is then imprinted in the probe absorption. As shown in Fig. 9, a clear peak at $0.25 \mathrm{PHz}$ is visible in Fourier power spectrum of the calculated hole density integrated around the amine group, in very good agreement with the pump-probe measurement. As discussed in [13], the sliding-window Fourier analysis of the numerical simulation, shown in Fig. 10, demonstrated that the main peak at $0.25 \mathrm{PHz}$ forms in about $15 \mathrm{fs}$ and disappears after about $35 \mathrm{fs}$, in agreement with the results of the Fourier analysis of the experimental data. A higher frequency component is visible around $0.36 \mathrm{PHz}$ in the delay intervals below $15 \mathrm{fs}$ and above $30 \mathrm{fs}$. The temporal evolution of the main Fourier components is a consequence of the complex interplay among several beating processes initiated by the
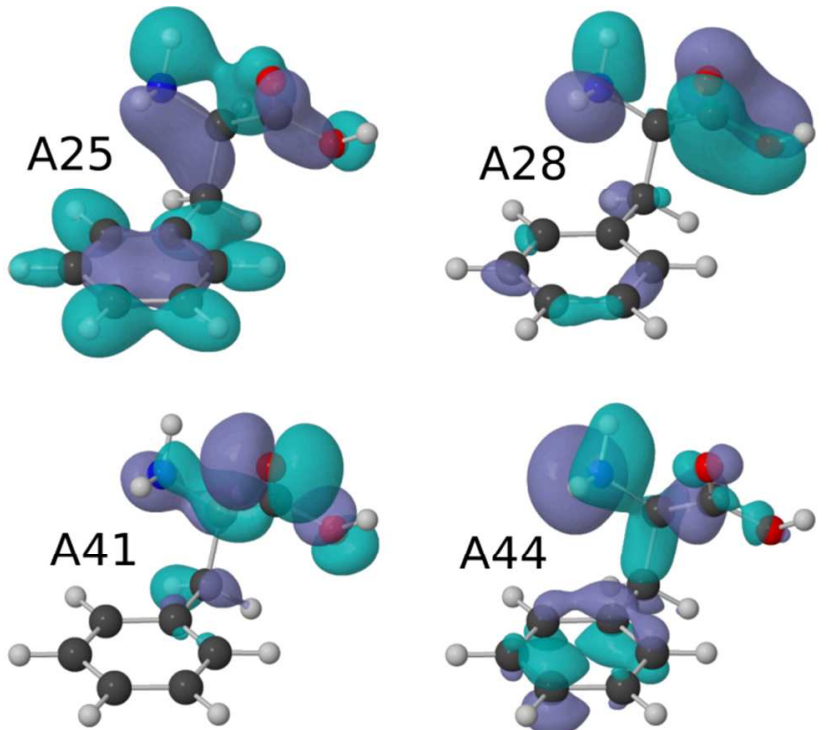

Fig. 12. Calculated Kohn-Sham orbitals of phenylalanine whose beating is mainly responsible of the Fourier peaks at $0.25 \mathrm{PHz}$ (A25-A28) and 0.36 $\mathrm{PHz}$ (A41-A44).
TABLE I

VIBRATIONAL FREQUENCIES OF THE HOMO-1 IONIC STATE OF PHENYLALANINE

\begin{tabular}{|c|c|c|c|}
\hline Mode & Wavenumber $\left(\mathrm{cm}^{-1}\right)$ & Freq. (PHz) & Period (fs) \\
\hline 1 & 34.7 & 0.0010 & 961.5 \\
\hline 2 & 55.2 & 0.0017 & 604.0 \\
\hline 3 & 68.1 & 0.0020 & 489.7 \\
\hline 4 & 92.6 & 0.0028 & 360.4 \\
\hline 5 & 164.7 & 0.0049 & 202.5 \\
\hline 6 & 218.5 & 0.0065 & 152.7 \\
\hline 7 & 281.7 & 0.0084 & 118.4 \\
\hline 8 & 339.8 & 0.0102 & 98.2 \\
\hline 9 & 365.6 & 0.0110 & 91.2 \\
\hline 10 & 384.7 & 0.0115 & 86.7 \\
\hline 11 & 395.2 & 0.0118 & 84.4 \\
\hline 12 & 409.4 & 0.0123 & 81.5 \\
\hline 13 & 486.0 & 0.0146 & 68.6 \\
\hline 14 & 503.6 & 0.0151 & 66.2 \\
\hline 15 & 515.5 & 0.0155 & 64.7 \\
\hline 16 & 554.4 & 0.0166 & 60.2 \\
\hline 17 & 596.9 & 0.0179 & 55.9 \\
\hline 18 & 631.1 & 0.0189 & 52.9 \\
\hline 19 & 652.6 & 0.0196 & 51.1 \\
\hline 20 & 676.3 & 0.0203 & 49.3 \\
\hline 21 & 782.3 & 0.0235 & 42.6 \\
\hline 22 & 791.3 & 0.0237 & 42.2 \\
\hline 23 & 805.8 & 0.0242 & 41.4 \\
\hline 24 & 833.5 & 0.0250 & 40.0 \\
\hline 25 & 841.8 & 0.0252 & 39.6 \\
\hline 26 & 913.8 & 0.0274 & 36.5 \\
\hline 27 & 970.2 & 0.0291 & 34.4 \\
\hline 28 & 999.9 & 0.0300 & 33.4 \\
\hline 29 & 1015.9 & 0.0305 & 32.8 \\
\hline 30 & 1026.7 & 0.0308 & 32.5 \\
\hline 31 & 1032.0 & 0.0309 & 32.3 \\
\hline 32 & 1070.2 & 0.0321 & 31.2 \\
\hline 33 & 1090.9 & 0.0327 & 30.6 \\
\hline 34 & 1109.3 & 0.0333 & 30.1 \\
\hline 35 & 1149.9 & 0.0345 & 29.0 \\
\hline 36 & 1172.1 & 0.0351 & 28.5 \\
\hline 37 & 1188.6 & 0.0356 & 28.1 \\
\hline 38 & 1207.6 & 0.0362 & 27.6 \\
\hline 39 & 1245.9 & 0.0373 & 26.8 \\
\hline 40 & 1286.9 & 0.0386 & 25.9 \\
\hline 41 & 1294.8 & 0.0388 & 25.8 \\
\hline 42 & 1341.3 & 0.0402 & 24.9 \\
\hline 43 & 1362.7 & 0.0409 & 24.5 \\
\hline 44 & 1370.8 & 0.0411 & 24.3 \\
\hline 45 & 1437.8 & 0.0431 & 23.2 \\
\hline 46 & 1470.8 & 0.0441 & 22.7 \\
\hline 47 & 1490.9 & 0.0447 & 22.4 \\
\hline 48 & 1509.2 & 0.0452 & 22.1 \\
\hline 49 & 1542.7 & 0.0462 & 21.6 \\
\hline 50 & 1610.1 & 0.0483 & 20.7 \\
\hline 51 & 1621.0 & 0.0486 & 20.6 \\
\hline 52 & 1832.2 & 0.0549 & 18.2 \\
\hline 53 & 3077.5 & 0.0923 & 10.8 \\
\hline 54 & 3093.9 & 0.0928 & 10.8 \\
\hline 55 & 3150.0 & 0.0944 & 10.6 \\
\hline 56 & 3173.8 & 0.0951 & 10.5 \\
\hline 57 & 3178.7 & 0.0953 & 10.5 \\
\hline 58 & 3188.6 & 0.0956 & 10.5 \\
\hline 59 & 3200.2 & 0.0959 & 10.4 \\
\hline 60 & 3206.1 & 0.0961 & 10.4 \\
\hline 61 & 3524.4 & 0.1057 & 9.5 \\
\hline 62 & 3674.4 & 0.1102 & 9.1 \\
\hline 63 & 3730.9 & 0.1118 & 8.9 \\
\hline
\end{tabular}

Wavenumber $\left(\mathrm{cm}^{-1}\right)$, frequency $(\mathrm{PHz})$ and corresponding periods (fs) of vibrational modes for the lowest cationic state of phenylalanine.

broad band excitation pulse. We note that the oscillation periods obtained from the experimental data and from the numerical simulations are shorter than the fastest vibrational 

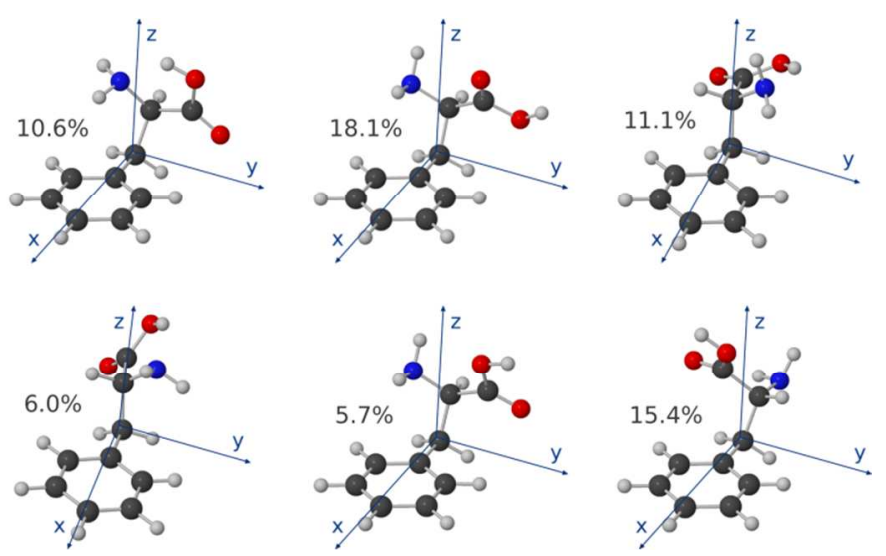

Fig. 13. Geometry of the most populated conformers of phenylalanine at the temperature of the experiment $(430 \mathrm{~K})$. Their relative populations are indicated as well as the 3 orthogonal orientations of the polarization vector of the electric field we have considered in the calculations.

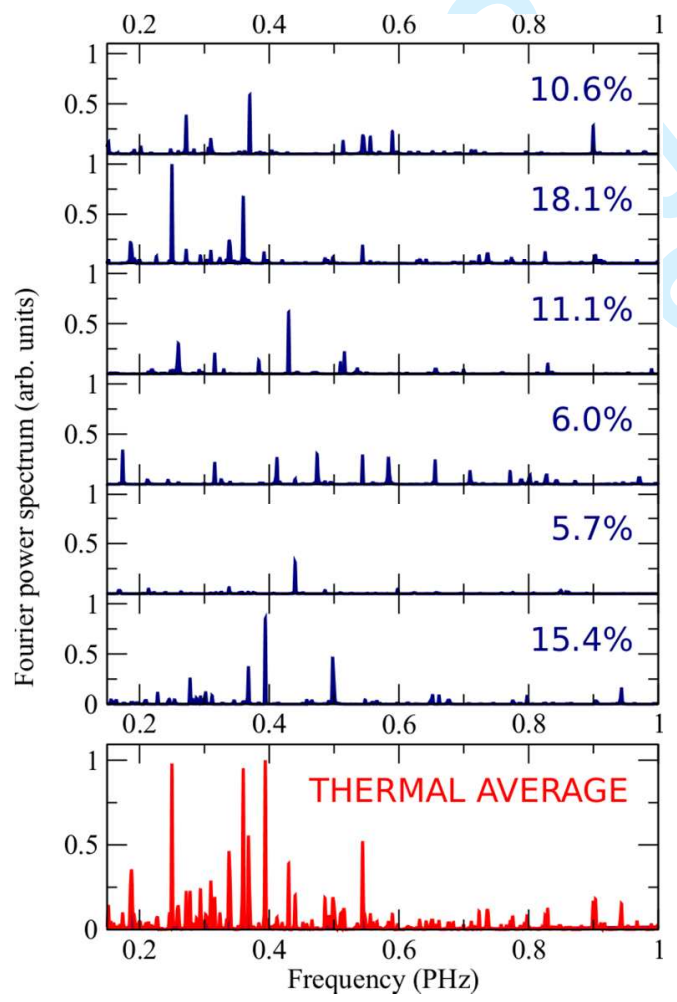

Fig. 14. Fourier power spectra of the hole density on the amine group for the most populated conformers, rescaled with their relative weights for a better illustration. The lower panel shows the averaged results.

period for the lowest cationic state of phenylalanine, which we have calculated by employing standard DFT-B3LYP quantum chemistry calculations (same procedure as for the geometry optimizations). The calculated vibrational frequencies are shown in Table I.

Of course we cannot claim that the relative intensities of the relevant beatings observed in the experiment can be explained by a theoretical calculation in which the probe step is not included (this may explain the discrepancies between measured and calculated peak intensities), but we can clearly identify that the observed beatings are unambiguously associated to charge fluctuations around the amine group, which in turn are associated to a small number of cationic states among all the accessible ones. Indeed, another important result of the numerical simulation is that it is possible to identify the ionic states that are responsible for the most important beatings. These are identified in Fig. 11, for the most abundant conformer. From the simulation it is possible to conclude that the main frequency peak around $0.25 \mathrm{PHz}$ originates from a beating between two states that contain a hole in a molecular orbital that is highly delocalized (A25) and a second one that is more localized on the amine group (A28), shown in Fig. 12. Similarly, the frequency component around $0.36 \mathrm{PHz}$ arises from an interference between two states (A41 and A44) that contain hole density on the amine and the carboxyl groups (see Fig. 12), which allows charge migration between the two sites. These interferences manifest as fluctuations in the electronic density on the amine group.

We have performed calculations for the 6 most populated conformers (see Fig. 13) at the temperature of the experiment (430K), according to previous theoretical work [46]. The corresponding Fourier spectra are shown in Fig. 14, together with the results for the thermal average (bottom panel in Fig. 14) obtained by taking into account the relative populations given in [46]. For the most populated conformers (and therefore for the averaged results), the frequencies at which the dominant peaks appear are in good agreement with those observed experimentally.

From the pump-probe measurements and the numerical simulations it is possible to conclude that charge fluctuations moving across the molecular chain, which ultimately lead to charge localization, are extremely fast even for a molecule as large as phenylalanine; therefore, charge localization (spontaneous or forced by external factors) can occur in a similar or even shorter time scale. Moreover, we have demonstrated that these fluctuations, which are required for an ulterior localization of the charge, can be induced by an attosecond pulse in spite of its broad bandwidth and, therefore, its low selectivity. The consequence is that one does not need to start from a localized charge state to induce charge fluctuations over the whole molecule (as done in most previous theoretical works). All this shows that attosecond pulses not only have the appropriate temporal resolution to study charge transfer, but also induce the necessary charge fluctuations that are needed to force localization in a particular molecular site (e.g., by using an external electromagnetic field). The only remaining issue is how to extract more precise information about this last step (localization) by using a probe pulse as the one employed in the present work or others. Certainly this requires further investigations from the experimental and theoretical points of view.

\section{CONCLUSION}

The observation of charge migration in complex molecular structures and, in particular, in biologically relevant molecules is one of the main targets of attosecond science. The investigation of charge evolution in these structures initiated by prompt ionization by attosecond pulses is important per se 
and for a better understanding of the interaction process between light and complex molecules. We adopted a novel methodology for the measurement of the charge migration process: we found that the yield of immonium dications is a particularly sensitive probe of charge location. Indeed, a clear ultrafast oscillation of the dication yield was measured, following the attosecond excitation, which we have unambiguously associated to the charge migration process within the investigated amino acid molecule. The results obtained in the case of phenylalanine can be seen as the first experimental confirmation that attosecond pulses and techniques are essential tools for understanding of dynamical processes on a temporal scale that is relevant for the evolution of crucial microscopic events at the heart of the macroscopic biological response of molecular complexes.

\section{REFERENCES}

[1] S. R. Leone, C. W. McCurdy, J. Burgdörfer, L. S. Cederbaum, Z. Chang, N. Dudovich, J. Feist, C. H. Greene, M. Ivanov, R. Kienberger, U. Keller, M. F. Kling, Z. -H. Loh, T. Pfeifer, A. N. Pfeiffer, R. Santra, K. Schafer, A. Stolow, U. Thumm, M. J. J. Vrakking, "What will it take to observe processes in 'real time'?," Nature Photon., vol. 8, no. 3, pp. 162-166, 2014.

[2] F. Krausz, M. Ivanov, "Attosecond physics," Rev. Mod. Phys., vol. 81, no. 1, pp. 163-234, 2009

[3] G. Sansone, M. Nisoli, "New frontiers in attosecond science," Progr. Quantum Electr., vol. 33, no. 1, pp. 17-59, 2009.

[4] A. H. Zewail, "Femtochemistry: Atomic-Scale Dynamics of the Chemical Bond," J. Phys. Chem. A, vol. 104, no. 24, pp. 5660-5694, 2000 .

[5] H. Gray, J. Winkler, "Electron tunneling through proteins," Q. Rev. Biophys., vol. 36, pp. 341-372, 2003.

[6] J. R. Winkler, H. B. Gray, T. R. Prytkova, I. V. Kurnikov, D. N. Beratan, "Electron transfer through proteins," in Bioelectronics, I. Willner, E. Katz, Eds. Weinheim: Wiley-VCH Verlag, 2005, pp. 15-33.

[7] F. Lépine, M. Y. Ivanov, M. J. J. Vrakking, "Attosecond molecular dynamics: fact or fiction?," Nature Photon., vol. 8, no. 3, pp. 195-204, 2014.

[8] G. Sansone, F. Kelkensberg, J. F. Pérez-Torres, F Morales, M.F. Kling, W. Siu, O.Ghafur, P. Johnsson, M. Swoboda, E. Benedetti, F. Ferrari, F Lépine, J.L. Sanz-Vicario, S. Zherebtsov, I. Znakovskaya, A. L'Huillier, M. Yu. Ivanov, M. Nisoli, F. Martín, M.J.J. Vrakking, "Electron localization following attosecond molecular photoionization," Nature, vol. 465 , no. 7299 , pp. 763-766, 2010.

[9] F. Kelkensberg, W. Siu, J. F. Pérez-Torres, F. Morales, G. Gademann, A. Rouzée, P. Johnsson, M. Lucchini, F. Calegari, J. L. Sanz-Vicario, F. Martín, M. J. J. Vrakking, "Attosecond Control in Photoionization of Hydrogen Molecules," Phys. Rev. Lett., vol. 107, no. 4, p. 043002, 2011.

[10] W. Siu, F. Kelkensberg, G. Gademann, A. Rouzée, P. Johnsson, D. Dowek, M. Lucchini, F. Calegari, U. De Giovannini, A. Rubio, R. R. Lucchese, H. Kono, F. Lépine, M. J. J. Vrakking, "Attosecond control of dissociative ionization of $\mathrm{O}_{2}$ molecules," Phys. Rev. A, vol. 84, no. 6, pp. 063412, 2011.

[11] P. Ranitovic, C. W. Hoglea, P. Rivière, A. Palaciosc, X.-M. Tong, N. Toshima, A. González-Castrillo, L. Martin, F. Martín, M. M. Murnane, H. Kapteyn, "Attosecond vacuum UV coherent control of molecular dynamics," PNAS, vol. 111, no. 3, pp. 912-917, 2014.

[12] L. Belshaw, F. Calegari, M. J. Duffy, A. Trabattoni, L. Poletto, M. Nisoli, J. B. Greenwood, "Observation of Ultrafast Charge Migration in an Amino Acid," J. Phys. Chem. Lett., vol. 3, no. 24, pp. 3751-3754, Dec. 2012.

[13] F. Calegari, D. Ayuso, A. Trabattoni, L. Belshaw, S. De Camillis, S. Anumula, F. Frassetto, L. Poletto, A. Palacios, P. Decleva, J.B. Greenwood, F. Martín, M. Nisoli, "Ultrafast electron dynamics in phenylalanine initiated by attosecond pulses," Science, vol. 346, no. 6207, pp. 336-339, Oct. 2014.

[14] L. S. Cederbaum, J. Zobeley, "Ultrafast charge migration by electron correlation," Chem. Phys. Lett., vol. 307, no. 3-4, pp. 205-210, 1999.

[15] F. Remacle, R. D. Levine, "An electronic time scale in chemistry," $P N A S$, vol. 103, no. 18, pp. 6793-6798, 2006.
[16] R. Weinkauf, P. Schanen, D. Yang, S. Soukara, E. W. Schlag, "Elementary processes in peptides: electron mobility and dissociation in peptide cations in the gas phase, "J. Phys. Chem., vol. 99, no. 28, pp. 11255-11265, 1995.

[17] R. Weinkauf, P. Schanen, A. Metsala, E. W. Schlag, M. Bürgle, H. Kessler, "Highly Efficient Charge Transfer in Peptide Cations in the Gas Phase: Threshold Effects and Mechanism," J. Phys. Chem., vol. 100, no. 47, pp. 18567-18585, Nov. 1996.

[18] S. Lünnemann, A. I. Kuleff, L. S. Cederbaum, "Charge Migration Following Ionization in Systems with Chromophore-Donor and AmineAcceptor Sites," J. Chem. Phys., vol. 129, no. 10, pp. 104305, 2008.

[19] S. Lünnemann, A. I. Kuleff, L. S. Cederbaum, "Ultrafast charge migration in 2-phenylethyl-N,N-dimethylamine," Chem. Phys. Lett., vol. 450 , no. 4-6, pp. 232-235, 2008.

[20] M. Nisoli, S. De Silvestri, O. Svelto, "Generation of high energy $10 \mathrm{fs}$ pulses by a new pulse compression technique," Appl. Phys. Lett., vol. 68, no. 20, pp. 2793-2795, 1996.

[21] M. Nisoli, S. De Silvestri, O. Svelto, R. Szipöcs, K. Ferencz, Ch. Spielmann, S. Sartania, F. Krausz, "Compression of high energy laser pulses below 5 fs," Opt. Lett., vol. 22, no. 8, pp. 522-524, 1997.

[22] A. Suda, M. Hatayama, K. Nagasaka, K. Midorikawa, "Generation of sub-10-fs, 5-mJ-optical pulses using a hollow fiber with a pressure gradient," Appl. Phys. Lett., vol. 86, no. 11, pp. 111116, 2005.

[23] C. Vozzi, F. Calegari, F. Ferrari, M. Lucchini, S. De Silvestri, O. Svelto, G. Sansone, S. Stagira, M. Nisoli, "Advances in laser technology for isolated attosecond pulse generation," Laser Phys. Lett., vol. 6, no. 4, pp. 259-267, 2009.

[24] T. Oksenhendler, S. Coudreau, N. Forget, V. Crozatier, S. Grabielle, R. Herzog, O. Gobert, D. Kaplan, "Self-referenced spectral interferometry," Appl. Phys. B, vol. 99, no. 1-2, pp. 7-12, 2010.

[25] F. Lücking, A. Trabattoni, S. Anumula, G. Sansone, F. Calegari, M. Nisoli, T. Oksenhendler, and G. Tempea, Opt. Lett., vol. 39, no. 8, pp. 2302-2305, 2014.

[26] F. Ferrari, F. Calegari, M. Lucchini, C. Vozzi, S. Stagira, G. Sansone, M. Nisoli, "High-energy isolated attosecond pulses generated by abovesaturation few-cycle fields," Nature Phot.,vol. 4, pp. 875-879, no. 12 , 2010.

[27] G. Sansone, L. Poletto, M. Nisoli, "High-energy attosecond light sources," Nature Photon., vol. 5, no. 11, pp. 655-663, 2011.

[28] Y. Mairesse, F. Quéré, "Frequency-resolved optical gating for complete reconstruction of attosecond bursts," Phys. Rev. A, vol. 71, no. 1, pp. 011401(R), 2005

[29] G. Sansone, E. Benedetti, F. Calegari, C. Vozzi, L. Avaldi, R. Flammini, L. Poletto, P. Villoresi, C. Altucci, R. Velotta, S. Stagira, S. De Silvestri, M. Nisoli, "Isolated Single-Cycle Attosecond Pulses," Science, vol. 314, no. 5798, pp. 443-446, 2006.

[30] L. Poletto, S. Bonora, M. Pascolini, P. Villoresi , "Instrumentation for analysis and utilization of extreme-ultraviolet and soft x-ray high-order harmonics," Rev. Sci. Instrum., vol. 75, no. 11, pp. 4413-4418, 2004.

[31] C. R. Calvert, L. Belshaw, M. J. Duffy, O. Kelly, R. B. King, A. G. Smyth, T. J. Kelly, J. T. Costello, D. J. Timson, W. A. Bryan, T. Kierspel, P. Rice, I. C. E. Turcu, C. M. Cacho, E. Springate, I. D. Williams, J. B. Greenwood, "LIAD-fs scheme for studies of ultrafast laser interactions with gas phase biomolecules," Phys. Chem. Chem. Phys., vol. 14, no. 18, pp. 6289-6297, 2012.

[32] S. Boyden and J. Zhang, "Temperature and Wavelength-Dependent Spectral Absorptivities of Metallic Materials in the Infrared," J. Therm. Heat Transfer, vol. 20, no. 1, pp. 9-15, 2006.

[33] D. Toffoli, M. Stener, G. Fronzoni, P. Decleva, "Convergence of the multicenter B-spline DFT approach for the continuum," Chem. Phys., vol. 276, no. 1, 25-43, 2002.

[34] R. van Leeuwen, E. J. Baerends, "Exchange-correlation potential with correct asymptotic behavior," Phys. Rev. A, vol. 49, no. 4, pp. 2421$2431,1994$.

[35] S. H. Vosko, L. Wilk, M. Nusair, "Accurate spin-dependent electron liquid correlation energies for local spin density calculations: a critical analysis," Can. J. Phys., vol. 58, no. 8, pp. 1200-1211, 1980.

[36] J. C. Slater, "Statistical Exchange-Correlation in the Self- Consistent Field," Adv. Quantum Chem., vol. 6, pp. 1-92, 1972.

[37] E. J. Baerends, D. E. Ellis, P. Ros, "Self-consistent molecular HartreeFock-Slater calculations I. The computational procedure," Chem. Phys., vol. 2, no. 1, pp. 41-51, 1973.

[38] C. Fonseca Guerra, J. G. Snijders, G. te Velde, E. J. Baerends, “Towards an order-N DFT method," Theor. Chem. Acc., vol. 99, no. 6, pp. 391403, 1998. 
[39] M. Stener, G. Fronzoni, P. Decleva, "Time dependent density functional study of the symmetry resolved $\mathrm{N} 1 \mathrm{~s}$ photoionization in $\mathrm{N}_{2}$," Chem. Phys. Lett., vol. 351, no. 5-6, pp. 469-474, 2002.

[40] D. Toffoli, M. Stener, G. Fronzoni, P. Decleva, "Convergence of the multicenter B-spline DFT approach for hethe continuum," Chem. Phys., vol. 276, pp. 25-43, 2002.

[41] C. Lee, W. Yang, R. G. Parr, "Development of the Colle-Salvetti correlation-energy formula into a functional of the electron density," Phys. Rev. B, vol. 37, no. 2, pp. 785-789, 1988.

[42] A. D. Becke, "Density-functional thermochemistry. III. The role of exact exchange," J. Chem. Phys., vol. 98, no. 7, pp. 5648, 1993.

[43] M. J. Frisch, G. W. Trucks, H. B. Schlegel, G. E. Scuseria, M. A. Robb, J. R. Cheeseman, G. Scalmani, V. Barone, B. Mennucci, G. A. Petersson, H. Nakatsuji, M. Caricato, X. Li, H. P.Hratchian, A. F. Izmaylov, J. Bloino, G. Zheng, J. L. Sonnenberg, M. Hada, M. Ehara, K. Toyota;R. Fukuda, J. Hasegawa, M. Ishida, T . Nakajima, Y. Honda, O. Kitao, H. Nakai, T. Vreven, J. A. MontgomeryJr., J. E. Peralta, F. Ogliaro, M. Bearpark, J. J. Heyd, E. Brothers, K. N. Kudin, V. N. Staroverov, R. Kobayashi, J. Normand, K. Raghavachari,A. Rendell, J. C. Burant, S. S. Iyengar, J. Tomasi, M.Cossi, N. Rega, J. M. Millam, M. Klene, J. E. Knox, J. B. Cross, V. Bakken, C. Adamo, J. Jaramillo, R. Gomperts, R. E. Stratmann, O. Yazyev, A. J. Austin, R. Cammi, C. Pomelli, J. W.Ochterski, R. L. Martin, K. Morokuma, V. G. Zakrzewski, G. A. Voth, P. Salvador, J. J. Dannenberg, S. Dapprich, A. D. Daniels, Ö. Farkas, J. B. Foresman, J. V. Ortiz, J. Cioslowski and D. J. Fox, GAUSSIAN09, Gaussian, Inc., Wallingford CT , Revised C01 edn., 2010 .

[44] K. Blum, Density Matrix Theory and Applications. Springer Series in Atomic, Optical and Plasma Physics, Berlin Heidelberg: SpringerVerlag, vol. 64, $3^{\text {rd }}$ Ed., 2012.

[45] N. Rohringer, R. Santra, "Multichannel coherence in strong-field ionization," Phys. Rev. A, vol. 79, no. 5, 053402, May 2009.

[46] Z. Huang, W. Yu, Z. Lin, "Exploration of the full conformational landscapes of gaseous aromatic amino acid phenylalanine: An ab-initio study," J. Mol. Struct. (Theochem), vol. 758, no. 2-3, pp. 195-202, 2006.

[47] O. Plekan, V. Feyer, R. Richter, M. Coreno, K. C. Prince, "Valence photoionization and photofragmentation of aromatic amino acids," Mol. Phys., vol. 106, no. 9-10, 1143-1153, 2008.

[48] S. Campbell, E. M. Marzluff, M. T. Rodgers, J. L. Beauchamp, M. E. Rempe, K. F. Schwinck, D. L. Lichtenberger, "Proton Affinities and Photoelectron Spectra of Phenylalanine and N-Methyl- and N,NDimethylphenylalanine. Correlation of Lone Pair Ionization Energies with Proton Affinities and Implications for N-Methylation as a Method to Effect Site Specific Protonation of Peptides," J. Am. Chem. Soc., vol. 116, no. 14, pp. 5257-5264, 1994.

[49] P. H. Cannington, N. S. Ham, "He(I) and He(II) photoelectron spectra of glycine and related molecules," J. Electron. Spectrosc. Relat. Phenom., vol. 32, no. 2, pp. 139-151, 1983.

[50] E. Goulielmakis, Z-H. Loh, A. Wirth, R. Santra, N. Rohringer, V.S. Yakovlev, S. Zherebtsov, T. Pfeifer, A.M. Azzeer, M.F. Kling, S.R. Leone, F. Krausz, "Real-time observation of valence electron motion," Nature, vol. 466, no. 7307, pp. 739-743, Aug. 2010.

[51] J. Breidbach, L. S. Cederbaum, "Migration of holes: formalism, mechanisms, and illustrative applications," J. Chem. Phys., vol. 118, no. 9, pp. 3983-3996, 2003.

[52] F. Martín, private communication.

[53] A. I. Kuleff, J. Breidbach, L. S. Cederbaum, "Multielectron wave-packet propagation: General theory and application," J. Chem. Phys., vol. 123, no. 4 , p. $044111,2005$.

[54] M. Stener, P. Bolognesi, M. Coreno, P. O'Keeffe, V. Feyer, G. Fronzoni, P. Decleva, L. Avaldi, A. Kivimäki, "Photoabsorption and S $2 \mathrm{p}$ photoionization of the SF6 molecule: resonances in the excitation energy range of 200-280 eV," J. Chem. Phys., vol. 134, no. 17, pp. 174311, 2011.

[55] D. Catone, M. Stener, P. Decleva, G. Contini, N. Zema, T. Prosperi, V. Feyer, K. C. Prince, S. Turchini, "Resonant circular dichroism of chiral metal-organic complex," Phys. Rev. Lett., vol. 108, no. 8, pp. 083001, 2012.

[56] L. Argenti, T. D. Thomas, E. Plesiat, X. -J. Liu, C. Miron, T. Lischke, G. Prumper, K. Sakai, T. Ouchi, R. Puttner, V. Sekushin, T. Tanaka, M. Hoshino, H. Tanaka, P. Decleva, K. Ueda, F. Martín, "Double-slit experiment with a polyatomic molecule: vibrationally-resolved $\mathrm{C} 1 \mathrm{~s}$ photoelectron spectra of acetylene," New J. Phys., vol. 14, no. 3, pp. 033012, 2012.
[57] S. Turchini, D. Catone, N. Zema, G. Contini, T. Prosperi, P. Decleva, M. Stener, F. Rondino, S. Piccirillo, K. C. Prince, M. Speranza, "Conformational Sensitivity in Photoelectron Circular Dichroism of 3Methylcyclopentanone," Chem. Phys. Chem., vol. 14, no. 8, pp. 1723$1732,2013$.

[58] R. K. Kushawaha, M. Patanen, R. Guillemin, L. Journel, C. Miron, M. Simon, M. N. Piancastelli and P. Decleva "From double-slit interference to structural information in simple hydrocarbons" PNAS, vol. 110, no. 38, pp. 15201-15206, 2013.

[59] K. Ueda, C. Miron, E. Plesiat, L. Argenti, M. Patanen, K. Kooser, D. Ayuso, S. Mondal, M. Kimura, K. Sakai, O. Travnikova, A. Palacios, P. Decleva, E. Kukk, F. Martín, "Intramolecular photoelectron diffraction in the gas phase," J. Chem Phys., vol. 139, no. 12, pp. 124306, 2013.

[60] D. M. P. Holland, D. A. Shaw, S. Coriani, M. Stener, P. Decleva, "A study of the valence shell electronic states of pyridazine by photoabsorption spectroscopy and time-dependent density functional theory calculations," J. Phys. B: At. Mol. Opt. Phys., vol. 46, no. 17, pp. 175103, 2013.

[61] E. Plesiat, P. Decleva, F. Martín, "Relationship between polarizationaveraged molecular-frame photoelectron angular distributions and geometry," Phys. Rev. A, vol. 88, no. 6, pp. 063409, 2013.

[62] R. Boll, D. Anielski, C. Bostedt, J. Bozek, L. Christensen, R. Coffee, S. De, P. Decleva, S. Epp, B. Erk, L. Foucar, J. Küpper, A. Rouzee, B. Rudek, A. Rudenko, S. Schorb, H. Stapelfeldt, M. Stener, S. Stern, S. Techert, S. Trippel, J. Ullrich, D. Rolles, "Femtosecond photoelectron diffraction on laser-aligned molecules: Towards time-resolved imaging of molecular structure," Phys. Rev. A, vol. 88, no. 6, pp. 061402, 2013.

[63] M. Stener, P. Decleva, T. Mizuno, H. Yoshida, A. Yagishita, "Offresonance photoemission dynamics studied by recoil frame F1s and $\mathrm{C} 1 \mathrm{~s}$ photoelectron angular distributions of $\mathrm{CH}_{3} \mathrm{~F}$, " J. Chem. Phys., vol. 140, no. 4 , pp. $044305,2014$.

[64] V. I. Lebedev, "Values of the nodes and weights of ninth to seventeenth order gauss-markov quadrature formulae invariant under the octahedron group with inversion," Zh. Vychisl. Mat. Mat. Fiz., vol. 15, no. 1, pp. 48-54, 1975.

[65] D. Dill, "Fixed-molecule photoelectron angular distributions," J. Chem. Phys., vol. 65, no. 3, pp. 1130-1133, 1976.

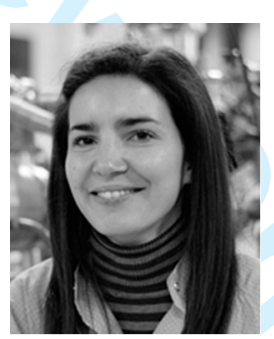

Francesca Calegari was born in Milan, Italy, in 1981. She received the Master's degree (cum laude) from Università degli Studi di Milano in 2005, and the Ph.D. from Politecnico di Milano in 2009. She was Postdoctoral Researcher with CNR-INFM until 2010 and with Politecnico di Milano until 2011. In 2010 she was a short-term visiting scientist at FOM institute AMOLF, Amsterdam, The Netherlands. Since 2012 she has a permanent position as a Researcher at CNR-IFN, Milan, and she is contract Professor of Physics at Politecnico di Milano. She is currently visiting scientist at MPSD-CFEL, Hamburg, Germany. She is coauthor of more than 50 research papers in international journals. Her current research interests include: attosecond science, ultrafast laser technology, control of electron dynamics in complex molecules. Dr. Calegari was awarded an European Research Council (ERC) Starting Grant in 2014.

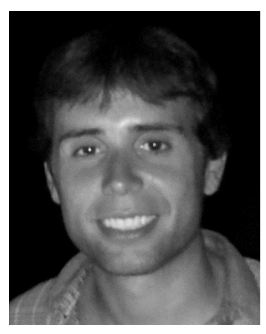

David Ayuso graduated in Chemistry at Universidad de Zaragoza in 2011. He received the Master's degree in Theoretical Chemistry and Computational Modeling at Universidad Autónoma de Madrid in 2013, investigating the interaction of small molecules with synchrotron radiation. He is currently working toward the Ph.D degree and his research is mainly focused on the theoretical description of vibrationally resolved photoionization processes and ultrafast electron dynamics in molecules initiated upon interaction with synchrotron radiation and/or attosecond pulses. 


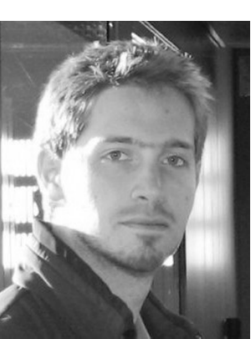

Andrea Trabattoni was born in Saronno, Italy, in 1987. He graduated in Theoretical Physics in 2011, investigating the symmetry properties of photonic quasicrystals. Since 2011 , he is a PhD student at the Department of Physics - Politecnico di Milano. His research activity is mainly dedicated to the development of attosecond laser sources and the application of attosecond technology to the investigation of ultrafast electron dynamics in complex systems, such as nanoparticles and biologically relevant molecules.

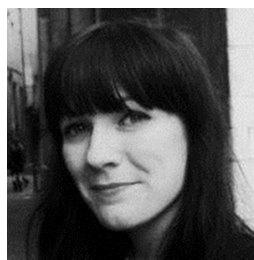

Louise Belshaw was born in Lisburn, Northern Ireland in 1988. She attained a MSci degree in Physics from the Queen's University Belfast in 2010 and a PhD in "Ultrafast Molecular Dynamics" from the same institution in 2013. She is currently training to become a Clinical Scientist in the Medical Physics Department of the Belfast Health and Social Care Trust.

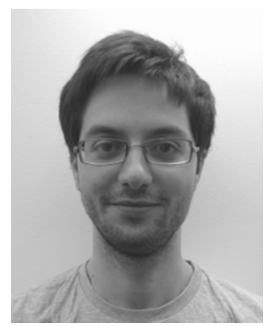

Simone De Camillis was born in Teramo, Italy, in 1988. He graduated with a Master's degree in Plasma Physics at University of Pisa, in 2013, investigating the generation of plasma instabilities in the solar wind-magnetosphere interaction region. Currently, he is studying for the $\mathrm{PhD}$ degree at Queen's University Belfast focusing on ultrafast intramolecular dynamics and charge transfers initiated by femtosecond or attosecond pulses.

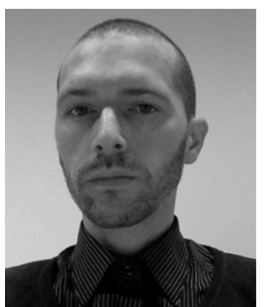

Fabio Frassetto was born in Treviso, Italy, in 1977. He received the Master's degree in Physics in 2002, and the Ph.D at the "Center of Studies and Activities for Space -G. Colombo-" in 2006, both from Università di Padova. After some Postdoctoral positions at the National Research Council Institute for Photonics and Nanotechnologies, CNRIFN of Padova, now he has a temporary position as researcher at CNR-IFN. He is author/co-author of more than 100 research papers/proceedings in international journals. His research activities are mainly focused on the design, realization and characterization of diagnostic instrumentation for Free Electron Laser facilities and High Harmonic Generation beam-lines for ultrafast experiments.

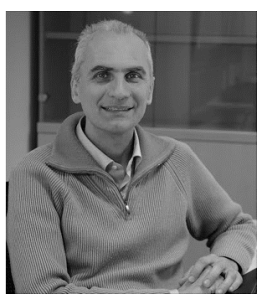

Luca Poletto was born in Vicenza, Italy, in 1968. He received the Master's degree (cum laude) in 1993 and the Ph.D. from University of Padova in 1996. From 1999 to 2006 he was Research Scientist of the National Institute for the Physics of Matter, Italy. Since 2007, he has been Senior Research Scientist of the Institute of Photonics and Nanotechnologies of the National Research Council (Italy). Since 2009, he has been the director of the Division of Padova of the Institute. He is co-author of more than 150 research papers in international journals. His research activities includes ultrafast optics in extreme-ultraviolet spectral region for synchrotron radiation, free-electron lasers and high-order laser harmonics; generation and handling of attosecond pulses; laser absorption spectroscopy for gas detection.

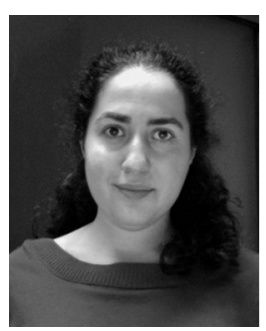

Alicia Palacios was born in Tomelloso, Spain, in 1979. She graduated in Chemistry in 2001 and received her $\mathrm{PhD}$ degree in 2006 at the Universidad Autónoma de Madrid. She completed her education with several stays in the Université Bordeaux I, and a long-term post-doctoral fellowship (2006-2009) in Lawrence Berkeley National Lab (Berkeley, CA). She is now an assistant professor at the Universidad Autónoma de Madrid. She has published more than 40 peer-reviewed articles in international journals working on atomic and molecular physics, in particular, developing new ab initio time-dependent treatments for the description of atoms and molecules subject to ultrashort intense laser pulses. She was awarded with a Marie Curie Integration Grant (ATTOTREND) as a Fellow Researcher.

Piero Decleva (Trieste, 1949) has spent his career at the Department of Chemistry, University of Trieste, where he became Lecturer in Theoretical Chemistry in 1976, Associate Professor of Quantum Chemistry from 1982 to 1994 and then Full Professor of Chemical Physics till present. He is author of 240 research papers in international journals. His research covered theoretical description of many-body effects and electron cross sections in molecular photoionization, with many collaborations with leading experimental groups. A large molecular code for the calculation of dynamical photoionization observables has been developed in Trieste, with recent emphasis on ultrashort time dependent phenomena and strong field ionization.

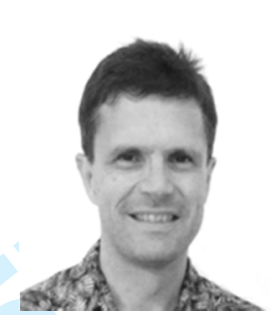

Jason Greenwood was born in Belfast, Northern Ireland, in 1970. He graduated with a Bachelor's degree from Oxford University in 1991 and a PhD in 1995 from Queen's University Belfast. After two years at NASA's Jet Propulsion Laboratory in Pasadena, California he returned to Queen's for a lectureship in 1999, becoming a Senior Lecturer in 2006. He is author of more than 70 research papers in international journals in areas including electronion and ion-atom/molecule collisions, X-ray emission from solar wind-cometary gas interactions, intense laser interactions with atoms and molecules, and ultrafast intramolecular dynamics.

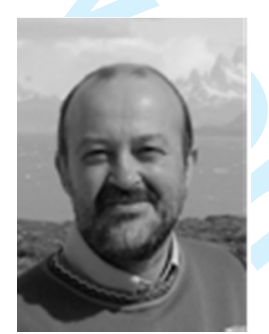

Fernando Martín was born in Madrid, Spain, in 1961. He graduated in Chemistry in 1984 and Physics in 1986 at the Universidad Autónoma de Madrid. He received his $\mathrm{PhD}$ Degree at the same university in 1986. He completed his postdoctoral studies at the University of Bordeaux (1988), the Université de Paris VI (1989-1990) and the Univesity of Chicago (1995-1996). He is Full Profesor at the Universidad Autónoma de Madrid since 2005. His research focuses on the theoretical modeling of photoexcitation and photoionization of atomic and molecular systems induced by synchrotron radiation and ultrashort laser pulses, as well as of complex molecular systems, isolated or deposited on surfaces. He has published over 350 articles and, since 2011, he is recipient of an Advanced Grant from the European Research Council.

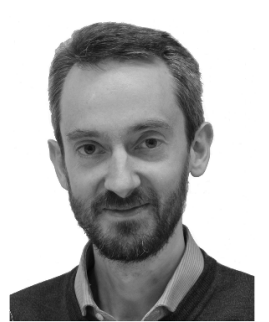

Mauro Nisoli was born in Bergamo, Italy, in 1965. From 1990 to 2000, he was a Researcher of the National Research Council (CNR) with the Center of Quantum Electronic and Electronic Instrumentation. From 2001 to 2010 he was an Associate Professor with the Department of Physics of Politecnico di Milano, where he is now Full Professor. He is author of more than 170 research papers in international journals, 1 patent, several invited and tutorial communications at international meetings and schools. His research focus are the following: attosecond science, ultrashort-pulse laser technology, control and real-time observation of electronic motion in atoms and molecules. Prof. Nisoli was awarded an European Research Council (ERC) Advanced Grant in 2009 